\title{
Global Regularity of 2D Generalized MHD Equations with Magnetic Diffusion
}

\author{
QUANSEN JiU1 \\ School of Mathematical Sciences, Capital Normal University \\ Beijing 100048, P. R. China \\ Email: jiuqs@mail.cnu.edu.cn \\ JIEFENG ZHAO \\ School of Mathematical Sciences, Capital Normal University \\ Beijing 100048, P. R. China \\ Email: zhaojiefeng001@163.com
}

\begin{abstract}
Abstract: This paper is concerned with the global regularity of the $2 \mathrm{D}$ (two-dimensional) generalized magnetohydrodynamic equations with only magnetic diffusion $\Lambda^{2 \beta} b$. It is proved that when $\beta>1$ there exists a unique global regular solution for this equations. The obtained result improves the previous known one which requires that $\beta>\frac{3}{2}$.
\end{abstract}

\section{Introduction}

Consider the Cauchy problem of the following two-dimensional generalized magnetohydrodynamic equations:

$$
\left\{\begin{array}{l}
u_{t}+u \cdot \nabla u=-\nabla p+b \cdot \nabla b-\nu \Lambda^{2 \alpha} u \\
b_{t}+u \cdot \nabla b=b \cdot \nabla u-\kappa \Lambda^{2 \beta} b \\
\nabla \cdot u=\nabla \cdot b=0, \\
u(x, 0)=u_{0}(x), \quad b(x, 0)=b_{0}(x)
\end{array}\right.
$$

for $x \in \mathbb{R}^{2}$ and $t>0$, where $u=u(x, t)$ is the velocity, $b=b(x, t)$ is the magnetic, $p=p(x, t)$ is the pressure, and $u_{0}(x), b_{0}(x)$ with $\operatorname{div} u_{0}(x)=\operatorname{div} b_{0}(x)=0$ are the initial velocity and magnetic, respectively. Here $\nu, \kappa, \alpha, \beta \geq 0$ are nonnegative constants and $\Lambda$ is defined by

$$
\widehat{\Lambda f}(\xi)=|\xi| \widehat{f}(\xi),
$$

\footnotetext{
${ }^{1}$ The research is partially supported by National Natural Sciences Foundation of China (No. 11171229, 11231006 and 11228102) and Project of Beijing Education Committee.
} 
where $\wedge$ denotes the Fourier transform. In the following sections, we will use the inverse Fourier transform $\vee$.

The global regularity of the 2D GMHD equations (1.1) has attracted a lot of attention and there have been extensive studies (see[4-[13]). It follows from [9] that the problem (1.1) has a unique global regular solution if

$$
\alpha \geqslant 1, \quad \beta>0, \quad \alpha+\beta \geqslant 2 .
$$

Tran, Yu and Zhai [7] got a global regular solution under assumptions that

$$
\alpha \geqslant 1 / 2, \beta \geqslant 1 \quad \text { or } \quad 0 \leqslant \alpha<1 / 2,2 \alpha+\beta>2 \quad \text { or } \quad \alpha \geqslant 2, \beta=0 .
$$

Recently, it was shown in [6] that if $0 \leqslant \alpha<1 / 2, \beta \geqslant 1,3 \alpha+2 \beta>3$, then the solution is global regular. In particular, when $\alpha=0, \beta>\frac{3}{2}$, the solution is global regular. This was proved independently in [11]12. Meanwhile, Fan, Nakamura and Zhou [4] used properties of the heat equation and presented a global regular solution when $0<\alpha<\frac{1}{2}, \beta=1$.

In this paper, we aim at getting the global regular solution of (1.1) when $\nu=0, \kappa>0$ and $\beta>1$. For simplicity, we let $\kappa=1$. That is, we consider

$$
\left\{\begin{array}{l}
u_{t}+u \cdot \nabla u=-\nabla p+b \cdot \nabla b \\
b_{t}+u \cdot \nabla b=b \cdot \nabla u-\kappa \Lambda^{2 \beta} b \\
\nabla \cdot u=\nabla \cdot b=0, \\
u(x, 0)=u_{0}(x), \quad b(x, 0)=b_{0}(x) .
\end{array}\right.
$$

Let $\omega=-\partial_{2} u_{1}+\partial_{1} u_{2}$ and $j=-\partial_{2} b_{1}+\partial_{1} b_{2}$ represent the vorticity and the current respectively. We will prove that $\omega, j \in L^{2}\left(0, T ; L^{\infty}\right)$ and obtain the global regularity of the solution by the BKM type criterion in [2]. To this end, we will take advantage of the approaches used in [4] and [6] to deal with the higher regularity estimates of $j$. More precisely, using the equation satisfied by the current $j$, we will obtain the estimates of $\left\|\Lambda^{r} j\right\|_{L^{2}}^{2}(t)+\int_{0}^{t}\left\|\Lambda^{\beta+r} j\right\|_{L^{2}}^{2} \leq C$ with $r=\beta-1$. Using the singular integral representation of $\Lambda^{\delta} j$ with some $\delta>0$, we will obtain the estimate $\|\nabla j\|_{L^{2}\left(0, T ; L^{\infty}\left(\mathbb{R}^{2}\right)\right)}$. Then we get the estimates of $\|\omega\|_{L^{2}\left(0, T ; L^{\infty}\left(\mathbb{R}^{2}\right)\right)}$ using the particle trajectory method. It should be noted that after the paper is finished, at the almost same time, Cao, Wu and Yuan obtain the similar result independently using a different method (see 3]). In comparison with result obtained in [3], it is not required that $\left\|\nabla j_{0}\right\|_{L^{\infty}}<\infty$ in our result.

The main result of this paper is stated as follows.

Theorem 1.1. Let $\beta>1$ and assume that $\left(u_{0}, b_{0}\right) \in H^{\rho}$ with $\rho>\max \{2, \beta\}$. Then for any $T>0$, the Cauchy problem (1.2) has a unique regular solution

$$
(u, b) \in L^{\infty}\left([0, T] ; H^{\rho}\left(\mathbb{R}^{2}\right)\right) \text { and } b \in L^{2}\left([0, T] ; H^{\rho+\beta}\left(\mathbb{R}^{2}\right)\right) .
$$

Remark 1.1. When $\alpha=0, \beta>\frac{3}{2}, \rho>2$, the result has been obtained in [6], [11] and [12].

\section{Preliminaries}

Let us first consider the following equation

$$
\left\{\begin{array}{l}
v_{t}+\Lambda^{2 \beta} v=f \\
v(x, 0)=v_{0}(x)
\end{array}\right.
$$

Similar to the heat equation, we can get

$$
v(x, t)=\int_{\mathbb{R}^{2}} t^{-\frac{1}{\beta}} h\left(\frac{x-y}{t^{\frac{1}{2 \beta}}}\right) v_{0}(y) \mathrm{d} y+\int_{0}^{t} \int_{\mathbb{R}^{2}}(t-s)^{-\frac{1}{\beta}} h\left(\frac{x-y}{(t-s)^{\frac{1}{2 \beta}}}\right) f(y, s) \mathrm{d} y \mathrm{~d} s,
$$


where $h(x)=\left(e^{-|\cdot|^{2 \beta}}\right)^{\vee}(x)$ and it has the similar properties as the heat kernel.

Lemma 2.1. Let $l$ be a nonnegative integer and $\eta \geqslant 0$, then

$$
\left\|\nabla^{l} h\right\|_{L^{1}}+\left\|\Lambda^{\eta} h\right\|_{L^{1}} \leqslant C \text {. }
$$

Proof. First, we give the proof of the estimates of $\nabla^{l} h$.

$$
\begin{aligned}
\left\|\nabla^{l} h\right\|_{L^{1}} & =C \sup _{|\gamma|=l} \int_{\mathbb{R}^{2}}\left|\int_{\mathbb{R}^{2}} \xi^{\gamma} e^{-|\xi|^{2 \beta}} e^{i x \cdot \xi} \mathrm{d} \xi\right| \mathrm{d} x \\
& =C \sup _{|\gamma|=l} \int_{|x| \leqslant 1}\left|\int_{\mathbb{R}^{2}} \xi^{\gamma} e^{-|\xi|^{2 \beta}} e^{i x \cdot \xi} \mathrm{d} \xi\right| \mathrm{d} x+C \int_{|x| \geqslant 1}\left|\int_{\mathbb{R}^{2}} \xi^{\gamma} e^{-|\xi|^{2 \beta}} e^{i x \cdot \xi} \mathrm{d} \xi\right| \mathrm{d} x \\
& \leqslant C+C \sup _{|\gamma|=l} \int_{|x| \geqslant 1}\left(1+|x|^{2}\right)^{-2}\left|\int_{\mathbb{R}^{2}} \xi^{\gamma} e^{-|\xi|^{2 \beta}}\left(1-\Delta_{\xi}\right)^{2} e^{i x \cdot \xi} \mathrm{d} \xi\right| \mathrm{d} x \\
& \leqslant C+C \sup _{|\gamma|=l} \int_{|x| \geqslant 1}\left(1+|x|^{2}\right)^{-2}\left|\int_{\mathbb{R}^{2}}\left(1-\Delta_{\xi}\right)^{2}\left(\xi^{\gamma} e^{-|\xi|^{2 \beta}}\right) e^{i x \cdot \xi} \mathrm{d} \xi\right| \mathrm{d} x \\
& \leqslant C .
\end{aligned}
$$

Next, we start to estimate $\Lambda^{\eta} h$ and let $l>\eta$.

$$
\begin{aligned}
\left\|\Lambda^{\eta} h\right\|_{L^{1}} & =\left\|\sum_{k \geqslant-1} \Delta_{k} \Lambda^{\eta} h\right\|_{L^{1}} \\
& \leqslant\left\|\Delta_{-1} \Lambda^{\eta} h\right\|_{L^{1}}+\sum_{k \geqslant 0}\left\|\Delta_{k} \Lambda^{\eta} h\right\|_{L^{1}} \\
& \leqslant C\|h\|_{L^{1}}+C \sum_{k \geqslant 0} 2^{k(-l+\eta)}\left\|\Delta_{k} \nabla^{l} h\right\|_{L^{1}} \\
& \leqslant C+C \sum_{k \geqslant 0} 2^{k(-l+\eta)}\left\|\nabla^{l} h\right\|_{L^{1}} \\
& \leqslant C,
\end{aligned}
$$

where we use the nonhomogeneous Littlewood-Paley decompositions $I d=\sum_{k} \Delta_{k}$ and BernsteinType inequalities (see[1]).

Now, let $\omega=\nabla^{\perp} \cdot u=-\partial_{2} u_{1}+\partial_{1} u_{2}$ and $j=\nabla^{\perp} \cdot b=-\partial_{2} b_{1}+\partial_{1} b_{2}$, and applying $\nabla^{\perp}$. to the equations (1.1), we obtain the following equations for $\omega$ and $j$ :

$$
\begin{aligned}
\omega_{t}+u \cdot \nabla \omega & =b \cdot \nabla j, \\
j_{t}+u \cdot \nabla j & =b \cdot \nabla \omega+T(\nabla u, \nabla b)-\Lambda^{2 \beta} j,
\end{aligned}
$$

where

$$
T(\nabla u, \nabla b)=2 \partial_{1} b_{1}\left(\partial_{1} u_{2}+\partial_{2} u_{1}\right)+2 \partial_{2} u_{2}\left(\partial_{1} b_{2}+\partial_{2} b_{1}\right) .
$$

The estimates for $\omega, j$ are obtained in [7] and [6], which is presented in the following lemma.

Lemma 2.2. Assume that $\alpha=0, \beta>1, r=\beta-1$, and $k \geqslant \beta$. Let $u_{0}, b_{0} \in H^{k}$. For any $T>0$, we have

$$
\begin{array}{r}
\|\omega\|_{L^{2}}^{2}(t)+\|j\|_{L^{2}}^{2}(t)+\int_{0}^{t}\left\|\Lambda^{\beta} j\right\|_{L^{2}}^{2} \mathrm{~d} \tau \leqslant C(T), \\
\left\|\Lambda^{r} j\right\|_{L^{2}}^{2}(t)+\int_{0}^{t}\left\|\Lambda^{\beta+r} j\right\|_{L^{2}}^{2} \mathrm{~d} \tau \leqslant C(T) .
\end{array}
$$




\section{The Proof of Theorem 1.1}

In this section, we will prove our main result Theorem 1.1. The proof is divided into three steps.

Step 1: $\omega \in L^{\infty}\left(0, T ; L^{p}\left(\mathbb{R}^{2}\right)\right), j \in L^{p}\left(0, T ; \mathbb{R}^{2}\right)$ for any $2<p<\infty$.

First, the second equation in (1.1) can be rewritten as

$$
b_{t}+\Lambda^{2 \beta} b=\sum_{i=1}^{2} \partial_{i}\left(b_{i} u-u_{i} b\right)
$$

Due to (2.3), we have

$$
b(x, t)=\int_{\mathbb{R}^{2}} t^{-\frac{1}{\beta}} h\left(\frac{x-y}{t^{\frac{1}{2 \beta}}}\right) b_{0}(y) \mathrm{d} y+\int_{0}^{t} \int_{\mathbb{R}^{2}}(t-s)^{-\frac{1}{\beta}} h\left(\frac{x-y}{(t-s)^{\frac{1}{2 \beta}}}\right) \sum_{i=1}^{2} \partial_{i}\left(b_{i} u-u_{i} b\right)(y, s) \mathrm{d} y \mathrm{~d} s .
$$

It follows from Lemma 2.2 that $b \in L^{\infty}\left(0, T ; L^{\infty}\left(\mathbb{R}^{2}\right)\right)$ and $u \in L^{\infty}\left(0, T ; L^{p}\left(\mathbb{R}^{2}\right)\right)$ for any $2 \leqslant p<\infty$. Thanks to Lemma 2.1, we can get

$$
\begin{aligned}
\|\nabla b\|_{L^{p}\left(0, T ; \mathbb{R}^{2}\right)} & \leqslant C(T)\left\|\nabla b_{0}\right\|_{L^{p}\left(\mathbb{R}^{2}\right)}+C\|b u\|_{L^{p}\left(0, T ; \mathbb{R}^{2}\right)} \int_{0}^{T}\left\|t^{-\frac{2}{\beta}}\left(\nabla^{2} h\right)\left(\frac{\cdot}{t^{\frac{1}{2 \beta}}}\right)\right\|_{L^{1}\left(\mathbb{R}^{2}\right)} \mathrm{d} t \\
& \leqslant C(T) \\
\left\|\nabla^{2} b\right\|_{L^{2}\left(0, T ; L^{p}\left(\mathbb{R}^{2}\right)\right)} & \leqslant C\left\|\nabla b_{0}\right\|_{L^{p}\left(\mathbb{R}^{2}\right)}\left(\int_{0}^{T}\left\|t^{-\frac{3}{2 \beta}}(\nabla h)\left(\frac{\cdot}{t^{\frac{1}{2 \beta}}}\right)\right\|_{L^{1}\left(\mathbb{R}^{2}\right)}^{2} \mathrm{~d} t\right)^{\frac{1}{2}} \\
& +C\|b \cdot \nabla u-u \cdot \nabla b\|_{L^{2}\left(0, T ; L^{p}\left(\mathbb{R}^{2}\right)\right)} \int_{0}^{T}\left\|t^{-\frac{2}{\beta}}\left(\nabla^{2} h\right)\left(\frac{\cdot}{t^{\frac{1}{2 \beta}}}\right)\right\|_{L^{1}\left(\mathbb{R}^{2}\right)} \mathrm{d} t \\
& \leqslant C(T)\left\|\nabla b_{0}\right\|_{L^{p}\left(\mathbb{R}^{2}\right)}+C(T)\|b \cdot \nabla u-u \cdot \nabla b\|_{L^{2}\left(0, T ; L^{p}\left(\mathbb{R}^{2}\right)\right)}
\end{aligned}
$$

for any $2 \leq p<\infty$.

Multiplying (2.5) by $|\omega|^{p-2} \omega(p>2)$, and integrating with respect to $x$, we get

$$
\begin{aligned}
\frac{1}{p} \frac{\mathrm{d}}{\mathrm{d} t}\|\omega\|_{L^{p}}^{p} & \leqslant \int_{\mathbb{R}^{2}}|b||\nabla j||\omega|^{p-1} \mathrm{~d} x, \\
& \leqslant\|b\|_{L^{\infty}}\|\nabla j\|_{L^{p}}\|\omega\|_{L^{p}}^{p-1}
\end{aligned}
$$

Thus, we have

$$
\frac{1}{2} \frac{\mathrm{d}}{\mathrm{d} t}\|\omega\|_{L^{p}}^{2} \leqslant\|b\|_{L^{\infty}}\|\nabla j\|_{L^{p}}\|\omega\|_{L^{p}}
$$

and

$$
\begin{aligned}
\|\omega\|_{L^{p}}^{2} & \leqslant C\|\omega(x, 0)\|_{L^{p}}^{2}+C \int_{0}^{t}\left(\|\nabla j\|_{L^{p}}^{2}+\|\omega\|_{L^{p}}^{2}\right) \mathrm{d} s \\
& \leqslant C+C \int_{0}^{t}\left(\left\|\nabla^{2} b\right\|_{L^{p}}^{2}+\|\omega\|_{L^{p}}^{2}\right) \mathrm{d} s \\
\frac{3.10}{\leqslant} & C+C \int_{0}^{t}\left(\|b \cdot \nabla u-u \cdot \nabla b\|_{L^{p}}^{2}+\|\omega\|_{L^{p}}^{2}\right) \mathrm{d} s \\
& \leqslant C+C \int_{0}^{t}\left(\|\nabla b\|_{L^{p}}^{2}\|u\|_{L^{\infty}}^{2}+\|\omega\|_{L^{p}}^{2}\right) \mathrm{d} s \\
& \leqslant C+C \int_{0}^{t}\left(1+\|\nabla b\|_{L^{p}}^{2}\right)\|\omega\|_{L^{p}}^{2} \mathrm{~d} s .
\end{aligned}
$$


This, combining with the Gronwall's inequality, leads to $\omega \in L^{\infty}\left(0, T ; L^{p}\left(\mathbb{R}^{2}\right)\right)$ for any $2<p<\infty$.

Step 2: $\nabla j \in L^{2}\left(0, T ; L^{\infty}\left(\mathbb{R}^{2}\right)\right)$.

Similar to [6], we apply $\Lambda^{\delta}(0<\delta<\min \{2 \beta-2, \rho-2\})$ on both sides of (2.6) to obtain

$$
\left(\Lambda^{\delta} j\right)_{t}+\Lambda^{2 \beta} \Lambda^{\delta} j=-\Lambda^{\delta}(u \cdot \nabla j)+\Lambda^{\delta}(b \cdot \nabla \omega)+\Lambda^{\delta}(T(\nabla u, \nabla b)) .
$$

Thanks to Lemma 2.2 and Step 1 , we have that $u j, b \omega$, and $T(\nabla u, \nabla b) \in L^{p}\left(0, T ; \mathbb{R}^{2}\right)$ for any $2<p<\infty$. In the same way as in Step 1, we have

$$
\begin{aligned}
\Lambda^{\delta} j(x, t) & =\int_{\mathbb{R}^{2}} t^{-\frac{1}{\beta}} h\left(\frac{x-y}{t^{\frac{1}{2 \beta}}}\right) \Lambda^{\delta} j_{0}(y) \mathrm{d} y \\
& +\int_{0}^{t} \int_{\mathbb{R}^{2}}(t-s)^{-\frac{1}{\beta}} h\left(\frac{x-y}{(t-s)^{\frac{1}{2 \beta}}}\right)\left(-\Lambda^{\delta}(u \cdot \nabla j)+\Lambda^{\delta}(b \cdot \nabla \omega)\right)(y, s) \mathrm{d} y \mathrm{~d} s \\
& +\int_{0}^{t} \int_{\mathbb{R}^{2}}(t-s)^{-\frac{1}{\beta}} h\left(\frac{x-y}{(t-s)^{\frac{1}{2 \beta}}}\right) \Lambda^{\delta}(T(\nabla u, \nabla b))(y, s) \mathrm{d} y \mathrm{~d} s
\end{aligned}
$$

and

$$
\begin{aligned}
\left\|\nabla \Lambda^{\delta} j\right\|_{L^{2}\left(0, T ; L^{p}\left(\mathbb{R}^{2}\right)\right)} & \leqslant C\left\|\Lambda^{\delta} j_{0}\right\|_{L^{p}\left(\mathbb{R}^{2}\right)}\left(\int_{0}^{T}\left\|t^{-\frac{3}{2 \beta}}(\nabla h)\left(\frac{\cdot}{t^{\frac{1}{2 \beta}}}\right)\right\|_{L^{1}\left(\mathbb{R}^{2}\right)}^{2} \mathrm{~d} t\right)^{\frac{1}{2}} \\
& +C\|u j\|_{L^{2}\left(0, T ; L^{p}\left(\mathbb{R}^{2}\right)\right)} \int_{0}^{T}\left\|t^{-\frac{4+\delta}{2 \beta}}\left(\Lambda^{\delta} \nabla^{2} h\right)\left(\frac{\cdot}{t^{\frac{1}{2 \beta}}}\right)\right\|_{L^{1}\left(\mathbb{R}^{2}\right)} \mathrm{d} t \\
& +C\|b w\|_{L^{2}\left(0, T ; l^{p}\left(\mathbb{R}^{2}\right)\right)} \int_{0}^{T}\left\|t^{-\frac{4+\delta}{2 \beta}}\left(\Lambda^{\delta} \nabla^{2} h\right)\left(\frac{\cdot}{t^{\frac{1}{2 \beta}}}\right)\right\|_{L^{1}\left(\mathbb{R}^{2}\right)} \mathrm{d} t \\
& +C\|T(\nabla u, \nabla b)\|_{L^{2}\left(0, T ; L^{p}\left(\mathbb{R}^{2}\right)\right)} \int_{0}^{T}\left\|t^{-\frac{3+\delta}{2 \beta}}\left(\Lambda^{\delta} \nabla h\right)\left(\frac{\cdot}{t^{\frac{1}{2 \beta}}}\right)\right\|_{L^{1}\left(\mathbb{R}^{2}\right)} \mathrm{d} t \\
& \leqslant C(T)
\end{aligned}
$$

for any $2<p<\infty$. So we can choose $\delta$ small and $p$ large enough such that $\nabla j \in L^{2}\left(0, T ; L^{\infty}\left(\mathbb{R}^{2}\right)\right)$ and $\left\|\Lambda^{\delta} j_{0}\right\|_{L^{p}} \leqslant C\left\|j_{0}\right\|_{H^{\rho}}$.

Step 3: $\omega \in L^{\infty}\left(0, T ; L^{\infty}\right)$.

Because of the estimates of the step 2, and the following equation

$$
\omega_{t}+u \cdot \nabla \omega=b \cdot \nabla j
$$

we can prove that $\omega \in L^{\infty}\left(0, T ; L^{\infty}\right)$ by using the particle trajectory method. By taking advantage of the BKM type criterion for global regularity (see [2]), we finish the proof of Theorem 1.1.

\section{References}

[1] H. Bahouri, J.Y. Chemin, R. Dachine, Fourier analysis and nonlinear partial differential equations, Springer, 2011

[2] R. E. Caflisch, I. Klapper, G. Steele, Remarks on singularities, dimension and energy dissipation for ideal hydrodynamics and MHD, Comm. Math. Phys. 184 (1997) 443-455

[3] C. Cao, J. Wu, B. Yuan, The 2D incompressible magnetohydrodynamics equations with only magnteic diffusion, (2013)ArXiv: 1306.3629 
[4] J. Fan, G. Nakamura, Y. Zhou, Global Cauchy problem of 2D generalized MHD equations, preprint.

[5] Q. Jiu, D. Niu, Mathematical results related to a two-dimensional magneto-hydrodynamic equations, Acta Math. Sci. Ser. B English. Ed. 26 (2006)744-756

[6] Q. Jiu, J. Zhao, A remark on global regularity of 2D generalized magnetohydrodynamic equations, (2013)ArXiv: 1306.2823

[7] C.V. Tran, X. Yu, Z. Zhai, On global regularity of 2D generalized magnetodydrodynamics equations, J. Differential. Equations. 254 (2013) 4194-4216

[8] J. Wu, Generalized MHD equations, J. Differential Equations. 195 (2) (2003) 284-312, 2003.

[9] J. Wu, Global regularity for a class of generalized magnetohydrodynamic equations, J. Math. Fluid Mech. 13 (2011) 295-305

[10] K. Yamazaki, On the global regularity of two-dimension generalized magnetohydrodynamics system, (2013) ArXiv: 1306.2842

[11] K. Yamazaki, Remarks on the global regularity of two-dimensional magnetohydrodynamics system with zero dissipation, (2013) ArXiv: 1306.2763

[12] B. Yuan, L. Bai, Remarks on global regularity of 2D generalized MHD equations, (2013) ArXiv: 1306.2190

[13] Y. Zhou, J. Fan, A regularity criterion for the 2D MHD system with zero magnetic diffusivity, J. Math. Anal. Appl. 378 (2011) 169-172 